\title{
Characterising sex differences of autosomal DNA methylation in whole blood using the Illumina EPIC array
}

\author{
Olivia A Grant ${ }^{1,2,3}$, Yucheng Wang ${ }^{1,4}$, Meena Kumari ${ }^{2}$, Nicolae Radu Zabet ${ }^{1,3,}$, Leonard Schalkwyk ${ }^{1, *}$ \\ ${ }^{1}$ School of Life Sciences, University of Essex, Colchester, CO4 3SQ, UK \\ ${ }^{2}$ Institute of Social and Economic Research, University of Essex, Colchester, CO4 3SQ, UK \\ ${ }^{3}$ Blizzard Institute, Barts and The London School of Medicine and Dentistry, Queen Mary University of \\ London, London, E1 2AT, UK \\ ${ }^{4}$ School of Computer Science and Electronic Engineering, University of Essex, CO4 3SQ, UK
}

To whom correspondence should be addressed. Tel: +44 0120687 2630; Fax: +44 0120687 2592. Email: r.zabet@qmul.ac.uk and lschal@essex.ac.uk

\begin{abstract}
Sex differences are known to play a role in disease etiology, progression and outcome. Previous studies have revealed autosomal epigenetic differences between males and females in some tissues, including differences in DNA methylation patterns. Here, we report for the first time an analysis of autosomal sex differences in DNAme using the Illumina EPIC array in human whole blood $(n=1171)$. We identified 554 sexassociated differentially methylated CpG sites (saDMPs) with the majority found to be hypermethylated in females (70\%). These saDMP's are enriched in CpG islands and CpG shores and located preferentially at 5'UTRs, 3'UTRs and enhancers. Additionally, we identified 311 significant sex associated differentially methylated regions (saDMRs). Transcription factor binding site enrichment revealed enrichment of transcription factors related to critical developmental processes and sex determination such as SRY and SOX9. Our study reports a reliable catalogue of sex associated CpG sites and elucidates several characteristics of these sites.
\end{abstract}

\section{INTRODUCTION}

Sex is an important covariate in all epigenetic research due to its role in the incidence, progression and outcome of many phenotypic characteristics and human diseases (1,2). There is an increasing interest as to which role epigenetic modifications (such as DNA methylation) may play in the underpinnings for relationships between environmental exposures and disease onset. In addition, sex has previously been shown to have a strong influence on DNA methylation variation (3-7). However, the idea that DNA methylation variation between males and females may underlie the sex biases observed in diseases has not been well documented thus far.

Sex differences in disease prevalence are sometimes explained at the molecular level and rooted in genetic differences between males and females. Differences in sex chromosome complement have independently been shown to direct differences in gene expression and chromatin organization (8-11). Furthermore, these differences in sex chromosome complement are sufficient to explain sex bias seen in some diseases. $X$ chromosome number has previously been shown to impact immune cell population and occasionally therefore the development of diseases such as autoimmunity $(12,13)$.

Previous research has also revealed sex differences in gene expression of autosomal genes as well as sex chromosome linked genes (14). It is worth noting that most of the differences in gene expression on the autosomes are small differences (15). However small expression differences may still be associated with great effects on phenotypic characteristics and disease incidence and onset. Others also identified sex 
differences in chromatin accessibility and histone modifications, thus suggesting that different epigenetic factors contribute to gene expression sex biases seen in some diseases (16).

Sex specific gene expression and levels of sex hormones may be mediated by epigenetic mechanisms, including DNA methylation. Several genome wide association methylome studies (or Epigenome Wide Association Studies - EWAS) have highlighted differences in DNA methylation patterns linked to sex differences in genes on the autosomes (17-20). Previous studies have reported sites showing varying methylation due to sex differences in various tissues such as saliva, placenta, brain, pancreatic islets and whole blood $(17,19,21-27)$. Early studies in this area did not account for cross hybridizing probes which map to both autosomal and sex chromosome loci $(28,29)$. A meta-analysis of 76 published studies with correction for cross hybridizing probes only validated 184 sex associated sites (30). Furthermore, cellular heterogeneity is also important for identification of sex associated sites as several studies have previously demonstrated that cell type proportions may occasionally vary by sex and that could result in identification of cell type associated type differences rather than sex associated differences (24).

Due to $\mathrm{X}$ chromosome inactivation in females, large differences in methylation levels of $\mathrm{X}$ chromosomes can be observed between males and females (31). Recent research suggests that normalising methylation data with the sex chromosomes introduces a large technical bias to many autosomal CpGs. This technical bias has been reported to result in many autosomal $\mathrm{CpG}$ sites being falsely associated with sex and extra steps need to be taken to ensure minimal technical bias is introduced to the autosomal CpGs.

Here, we use the EPIC BeadChip to assess autosomal sex differences in DNA methylation levels from whole blood at individual sites and genomic regions. All individuals involved in this study were part of Understanding Society: The UK Household longitudinal study (32). Additionally, we account for cell type composition, cross hybridization and adequately handle the technical bias introduced by sex chromosomes. To our knowledge, this is the first study using the Illumina EPIC BeadChip (allowing for interrogation of $\sim 850,000$ sites across the genome) to investigate autosomal sex differences in DNA methylation.

\section{MATERIAL AND METHODS}

\section{Participants}

Whole blood Illumina Infinium MethylationEPIC BeadChip DNAme data was collected from 1175 participants involved in Understanding Society: The UK Household Longitudinal Study (33). In wave 3 of the study (201112) blood samples were collected from a portion of the study participants. Individuals were considered eligible to give a blood sample if they were over the age of 16 and met the other requirements detailed in the UK Household Longitudinal Study Biomarker user guide. Our study population was restricted to participants of white ethnicity. A full description of the dataset and data processing has been described by (34).

\section{DNA methylation data}

Samples of whole blood DNA from 1175 participants were obtained following the protocol described in (34). Raw signal intensities were processed using the R package bigmelon (35) and wateRmelon (36)from idat files. Prior to normalisation of the data, outlier samples were identified using principal component analysis and subsequently removed from the data set. The reported age of each sample was compared to predicted age using the epigenetic age method implemented by agep in the R package bigmelon (35) .Further, the reported sex of the samples was checked using a DNA methylation-based sex classifier (31)which predicts sex based on the methylation difference of $X$ and $Y$ chromosomes. 4 samples were subsequently removed after this step as reported and predicted sex did not match. The data was then normalised via the interpolated $X Y$ adjusted dasen method using the adjustedDasen function in the R package watermelon (37) which first normalises autosomal CpGs by dasen and then the corrected methylation values of sex chromosome linked CpGs are estimated as the weighted average of their nearest neighbours on autosomes. Following normalisation of the data, SNP probes, cross hybridizing probes (29) and $X$ or $Y$ linked probes 
were removed from the data set. The final data set consisted of 1171 samples and 747,361 DNA methylation sites.

To ensure that whole blood cell composition did not differ significantly by sex and would not introduce bias to our results, the relative proportions of Granulocytes, mononuclear, natural killer, CD4T, CD8T and B cells were estimated for all samples using the estimateCellCounts function implemented in bigmelon (35). Furthermore, to assess whether the sex differences we observed were age independent, we performed a Mann-Whitney $U$ test between the age distribution of males and females. Our results confirmed that there is no statistical difference in age between our male and female samples ( $p$ value 0.07 ; median values of 60 and 58 , respectively).

\section{Identifying sex associated autosomal differential methylation.}

Sex associated autosomal differentially methylated positions (saDMPs) were identified by performing linear modelling using the ChAMP package in R (38) using sex and Beta values. Correction for multiple testing was performed with the Benajamini-Hochberg false discovery rate method (FDR values). A probe was considered significantly differentially methylated if the difference in Beta values between males and females was greater than 0.05 in either direction and the FDR value was smaller than 0.05 . We further characterized differentially methylated regions (DMRs) by applying the DMRcate function from the R package ChAMP (38) to detect DMRs between males and females on the autosomes. A DMR was considered to be significantly associated with sex (saDMR) if the FDR value was smaller than 0.05 and consisted of at least $2 \mathrm{CpG}$ sites with a difference in beta values between males and females greater than 0.05 .

\section{Genomic annotation of CpG sites}

We annotated the autosomal CpG's using the manufacturer supplied annotation data (MethylationEPIC_v-10_B2 manifest file). Annotation was completed in the R package Minfi (39). Several categories were used as annotations in relation to $\mathrm{CpG}$ islands and divided into the following categories: CGls, CGI shores (S and N), $\mathrm{CGI}$ shelfs ( $\mathrm{S}$ and $\mathrm{N}$ ) and open sea regions. Further, we also annotated the autosomal $\mathrm{CpGs}$ to several genomic features, including exons, introns, 5' UTR, 3'UTR, enhancers, promoters and transposable elements (TEs) using data from UCSC table browser (https://genome.ucsc.edu/cgi-bin/hgTables).

\section{Gene ontology analyses}

GO analyses were conducted using the gometh function in the missMethyl package (40) which tests gene ontology enrichment for significant CpGs while accounting for the differing number of probes per gene present on the EPIC array. For GO ontology analyses of enriched TFBS we used enrichGO from the clusterProfiler package in $\mathrm{R}$ (41), which performs FDR adjustment.

\section{Enrichment of saDMPs in transcription factor binding motifs and integration with gene expression}

The enrichment analysis of known motifs in sex associated DMPs was performed using the $R$ package PWMEnrich (42) using the MotifDb collection of TF motifs (43). Specifically, the DNA sequences within a 100 bp range from the saDMP which were hypermethylated in females were extracted from the genome and compared to the saDMPs which were hypermethylated in males as the background to reveal unique enriched motifs (adjusted p-value < 0.05). RNA-seq data for 20 healthy donors ( 10 males and 10 females) from publicly available data from GEO (GSE120312) was used in our analysis. In particular, we used the preprocessed count matrices with DESeq2 (44) to calculate differentially expressed genes between males and females with an adjusted p-value of 0.05 and $\log _{2}$ fold change of 1 .

\section{Overlap of saDMP's with chromatin loops}

We examined whether any of the sex associated DMPs made 3D contacts with distal genes using $\mathrm{Hi}-\mathrm{C}$ data available from the Gene Expression Omnibus (GEO) under accession number (GSE124974) for white blood cells and neutrophils. Hi-C library preparation was performed using the Arima-HiC kit and pre-processing of the data was performed using Juicer command line tools (45). Reads were aligned to the human (hg38) genome using BWA-mem (46) and then pre-processed using the Juicer pre-processing pipeline. We called 
chromatin loops using the HICCUPS tool from Juicer using a $10 \mathrm{~Kb}$ resolution. We then constructed Genomiclnteractions objects to annotate saDMPs to loop anchors using the findOverlaps function from the GenomicRanges package using a maxgap of 10000. Following this, we then annotated the corresponding anchor to the relevant gene ID. These steps then allowed us to perform network analysis in Cytoscape (47) and GO and KEGG analyses in clusterProfiler (41).

\section{Protein-protein network visualisation and hub gene identification}

We searched all of the genes annotated to our saDMPs using the Search Tool for the Retrieval of Interacting Genes (STRING) (https:://string-db.org) database to generate our networks. We extracted protein-protein interactions with a combined score of $>0.4$. Then, we extracted top 50 genes using degree of connectivity methods in the plugin tool CytoHubba. The same analysis was repeated for the enriched transcription factor motifs found at saDMPs.

\section{RESULTS}

\section{Females show higher methylation at a subset of autosomal loci}

Analysis of DNA methylation (DNAme) differences between males and females on the autosomes was performed using linear regression for the IlluminaEPIC BeadChip for 1171 individuals (682 females and 489 males). After data processing and cleaning, $n=747,302$ CpGs were analysed (see Material and Methods). Sites which are known SNP probes, cross hybridizing or X/Y linked probes were excluded. After adjusting for multiple testing using the Benjamini Hochberg FDR method (FDR $p<0.05$ ) we identified 14,653 CpGs associated with sex. Of those CpGs, 68\% (9960 CpGs) were more highly methylated in females and the remaining 32\% (4693 CpGs) were more methylated in males. Gene ontology analyses showed several enriched terms for these 14,653 CpGs (Table 1) which included many terms related to development such as cellular developmental processes, system development, anatomical structure development and multicellular organism development. Furthermore, other enriched terms included plasma membrane, ion transport and more (see Table 1).

The lambda value of the Q-Q plot is 1.99 (Figure S1A) indicating slight inflation of test statistics and, in order to ensure we detect true sex differences, we selected CpGs that displayed large differences in methylation. Thus, we further filtered our list of $14,653 \mathrm{CpG}$ by only considering those probes that displayed a $\Delta$ Beta value (absolute difference between average Beta values in male and female samples) greater than 0.05 . A total of $554 \mathrm{CpGs}$ met this criterion (called sex associated DMPs or saDMPs) and were distributed across all autosomes (Figure 1A). $70 \%$ of the saDMPs were hypermethylated in females (389 CpGs) and $30 \%$ of which were hypermethylated in males (166 CpGs) (Figure 1B) (See Additional File 1 for the full list). Moreover, since whole blood is a bulk tissue, we calculated the estimated cell type proportions for whole blood between our male and female samples to assess whether any differences in cell type proportions would potentially be reflected in our results resulting in false positives. Using Wilcoxon test, we found no significant difference in the proportions of Granulocytes and CD4T cells between males and females, but we did find statistically significant differences in proportions of CD8T, Natural killer, B cells and monocytes (Supplementary figure 1B). We therefore performed our analysis with and without accounting for cell type proportions to see how this would affect our results. We found that we still retrieved the same list of saDMPs with and without accounting for cell type proportions allowing us to conclude that differences in cell type proportions did not affect our analysis. 
Since we had such stringent parameters to define what we considered a significantly associated saDMP for males and females. We performed principal component analysis (PCA) to see how male and female beta values clustered in PC space and to evaluate the effect of DNAme at the saDMPs. As shown in Figure 1C, male and female samples formed clear clusters based on the beta values of the significant sex associated DMPs (554 CpGs). PC1 explained $14.2 \%$ of the variance and PC2 explained $4.1 \%$ of the variance. Based on Figure $1 \mathrm{C}$ we can conclude that these saDMPs are sufficient to contribute to the clear separation of male and female samples in PC space.

\section{Characterisation of sex associated DMPs}

The saDMPs were found in 223 unique genes with 68 of these genes harbouring several saDMPs with an average of 2.7 saDMPs per gene (Figure 1D). CRISP2, a gene known to be involved in sperm function and male fertility (Lim et al.2019), harboured the largest number of saDMPs, 9. We performed GO and KEGG analyses, but did not identify any significantly enriched biological processes or pathways for these genes.

To help us try to gain more insight into the functional role of these saDMPs, we characterised their genomic location and further compared this with the autosomal background. We found saDMPs are preferentially located $\mathrm{CpG}$ islands and $\mathrm{CpG}$ shores and depleted in open sea regions compared to the autosomal background (Figure 1E). Moreover, saDMPs hypermethylated in females are enriched at promoters and exons, with saDMPs hypermethylated in males being enriched at 5'UTR, potentially acting as alternative promoters (Figure 1F). Interestingly, we observed that all saDMPs display enrichment at enhancers, which, together with their presence at promoters, indicates they could play a role in gene regulation. Lastly, we also note that all saDMPs were depleted at transposable elements and introns compared to the autosomal background.

Enrichment of saDMPs at enhancers suggests that some of the saDMPs could potentially regulate distal genes $(48,49)$. We further annotated the saDMPs to genes by assessing their contacts with promoters, and thus, distal genes. Following this, we further annotated the saDMPs to 46 additional genes, 35 of them being annotated to saDMPs hypermethylated in females and 11 to saDMPs hypermethylated in males (see Figure S2A-B).

To evaluate the interactions between the proximal (located in genes) and distal (those in 3D proximity) saDMPs, we collated these and produced protein-protein interaction networks to visualize the networks of these genes (see Figure S2C-D). Of the 11 genes linked to saDMPs hypermethylated in males, we found three histones (HIST1H3A, HIST1H4A and HIST1H4B), which are known to interact with CDYL (Figure S2C), a gene that we also found to harbour an saDMP hypermethylated in males. Chromodomain $Y$-like protein (CDYL) is a chromatin reader binding to heterochromatin (H3K9me3, H3K27me2 and H3K27me3) that is crucial for spermatogenesis, male fertility and $X$ chromosome inactivation (50). From the list of genes linked to saDMPs hypermethylated in females, KDM2A regulates circadian gene expression by repressing activity of CLOCK-ARNTL which has previously been shown to exhibit sex dimorphism (51). In addition, ODF2L; outer dense fiber of sperm tails 2 like is also linked to saDMPs hypermethylated in females and has previously be shown to interact with PRSS23, which is involved in ovulation (52). 
We then performed functional enrichment of genes annotated to those saDMPs to identify enriched biological processes or terms. Enrichment of the genes annotated to those saDMPs hypermethylated in females revealed several processes (129 terms), including adaptive immune system, estrogen receptor binding and androgen receptor binding (FDR < 0.05) (Additional File 5). Furthermore, enrichment of the genes annotated to those saDMPs hypermethylated in males displayed enrichment of terms such as dosage compensation by inactivation of $\mathrm{X}$ chromosome, hormone receptor binding and gene / chromatin silencing $($ FDR $<0.05)$ (Additional File 6).

\section{Enrichment of saDMPs in transcription factor binding sites}

To identify common features among the sex associated DMPs, we performed transcription factor (TF) binding site and gene ontology analyses. First, we evaluated whether the saDMPs were enriched in motifs for TFs (100 bp window). For the saDMPs hypermethylated in females, we found 329 enriched TFs (p.value $<0.05$ ) (Figure 2A and Additional File 3) with strongest evidence for TROVE2, XRCC1 and NFIL3. TROVE2/R060 is an RNA binding protein known to be involved in stimulating of androgen receptor regulated genes. We also found SOX9 and SRY TFs to be enriched in DMPs hypermethylated in females, which are genes known to be involved in male sex determination (Figure 2A) (53). For saDMPs hypermethylated in males, we identified 64 enriched TFs, including CEPBG, AFF4 and ELK1. CEPBG is a transcription factor which has previously been reported to stimulate adipocyte differentiation in an ESR1/CEBPA mediated pathway (54). Furthermore, GABPA and ELK1 have previously been shown to be associated with sex associated differentially methylated regions in the mouse liver (55).

To analyse whether the TF motifs were enriched for annotation to biological processes or pathways, we performed pathway analyses using the GO and KEGG databases. We identified 15 enriched KEGG pathways for the TFBS enriched at saDMPs hypermethylated in females, spanning a wide range of processes such as the transcriptional regulation in cancer, several specific cancer pathways, viral carcinogensis and more (Figure 2B). In addition, we also found 39 enriched GO terms ranging from transcription factor activity, E-box binding, transcription coactivator activity and interestingly, bHLH transcription factor binding (Figure S3B). Nevertheless, we found no enriched KEGG terms for the TFs enriched at saDMPs hypermethylated in males, likely due to the small number of enriched TFs. However, we identified 9 enriched GO terms such as NAD, NADP binding and oxidoreductase activity (Figure 3SA).

One possibility is that transcription factor motifs for genes encoded on the sex chromosomes may act as hubs in the enriched TF motif network. To assess this, we produced protein-protein interaction networks to visualize the networks of these TFs (Figure S4). Although we identified some enriched motifs for several TFs encoded on the $X$ chromosomes in the saDMPs hypermethylated in males such as ELK1, TGIF2LX and TCEAL6 (Figure S4A), we observed that they were not central hub nodes in the network. Nevertheless, we did identify several central TF motifs encoded on the sex chromosomes for the saDMPs hypermethylated in females (Figure S4B). These included 11 TFs encoded on the $X$ chromosome and 2 on the $Y$ chromosome including SRY and KDM5D.

We further utilised cytohubba package to robustly identify if these TFs were in fact hub genes in the network. This revealed that ELK1 did in fact act as a hub gene in the TF network, however the other 49 genes were encoded on the autosomes (Figure 2C). Interestingly, for the TF motifs enriched at saDMPs hypermethylated 
in females, we found SRY (coded for on the Y chromosome) and SOX9, BRCA1 and other autosomal genes (Figure 2D). BRCA1 is a tumour suppressor gene which is heavily implicated in breast cancer and its promoter hypermethylation in peripheral blood has previously been shown to be linked to decreased risk of breast cancer (56).

\section{Relationship with gene expression}

The 554 saDMPs were then further explored in association with the expression levels of their annotated genes using publicly available data for whole blood poly $(A)+$. We did not identify any sex biased gene expression patterns corresponding to differences in DNAme levels at these genes (Figure S5). Some of the genes annotated to our saDMPs met the $P$ value threshold after correcting for multiple testing using the Benjamini and Hochberg method (adjusted pvalue<0.05), however did not meet the log fold change threshold $\left(\log _{2} \mathrm{FC}>1\right)$ such as TARP, KCNT1 and CEBPB (Figure S5A). However, these genes have low expression in blood and therefore, this analysis may be more relevant for other tissues such as brain or skeletal muscle. The majority of the differentially expressed genes are located on the sex chromosomes, but we also did observe differential expression between males and females for several autosomal genes (Figure S5B-S5C). These results indicate that the differences in DNA methylation observed between males and females do not lead to any large difference in gene expression, but only some small ones.

\section{Sex associated differentially methylated regions}

Given that several genes harboured numerous saDMPs, we postulated whether some of the saDMPs were part of larger differentially methylated regions associated with sex. We therefore searched for differentially methylated regions associated with sex. Following adjustment for multiple testing (FDR), we identified a large list of 14,386 sex associated differentially methylated regions. We therefore considered a saDMRs significant if it harboured at least $2 \mathrm{CpGs}$, had an FDR value smaller than 0.05 and had a maximum absolute beta fold change value within the region greater than 0.05 in either direction. Following filtering of the list of saDMRs, we identified 311 significant sex associated DMRs on the autosomes between males and females located in 226 unique genes (Additional File 2). The number of CpGs within the DMRs ranged from 3 to 58 and had an average width of 1793 base pairs (bp) ranging from 63bp to $5638 \mathrm{bp}$.

Figure 3 shows the methylation values for males and females at 4 of the most significant saDMRs: (i) the strongest saDMR (31 CpGs) overlaps ATP5J and GABPA genes on chromosome 21 (Figure 3A), (ii) the second most significant saDMR (12 CpGs) overlaps DYRK2 gene on chromosome 12 (Figure 3B), (iii) the third strongest DMR (12 CpGs) overlaps SMAD2 gene on chromosome 18 (Figure 3C) and (iv) the fourth strongest saDMR (10 CpGs) overlaps PRKXP1 pseudogene on chromosome 15 (Figure 3D). DYRK2 and SMAD2 are hypermethylated in females, while ATP5J, GABPA and PRKXP1 are hypermethylated in males. Note that PRKXP1 saDMR, although hypermethylated in males, contains a single CpG which is hypermethylated in females.

A saDMR harbouring $58 \mathrm{CpGs}$ overlapped the promoter region of a gene called SCAND3. The top hits in the saDMR list overlapped promoter regions of the following genes; ATP5J, GABPA, DYRK2, SMAD2, CRISP2, AND PRKXP1. ATP5J and GABPA are genes (hypermethylated in males) which have previously been reported to be implicated in early onset of Alzheimers disease (57), a disease known to affect females more 
than males. Furthermore, ATP5J is a gene known to be a target gene of oestrogen, previously shown to serve an inhibitory role in the sex differences in hepatocellular carcinoma (58). DRYK2, SMAD2 and CRIPS2 have previously been shown to exhibit functions which are sex specific (21). CpGs harboured by the gene ZPBP2 are hypermethylated in females and previous work has implicated this gene in sex specific effects in asthma and, consistent with our findings, also showed that methylation at this gene is lower in males than females. PRKXP1 is located on chromosome 15 and CpGs in this region have previously been associated with Crohns disease and intestinal inflammation, a disease which has previously been reported to be more prevalent in females (59). These results suggest that DNAme differences at these sites may translate to sex biases seen in disease such as Alzheimers and asthma. Moreover, SCAND3 is expressed mainly in seminal vesicle and testis, CRIPS2 is expressed uniquely in testis, whereas GABPA has high expressions in placenta and vagina.

\section{DISCUSSION}

Here, we conducted the first study aiming to characterize autosomal sex differences in DNAme between males and females in whole blood using the IlluminaEPIC BeadChip, which interrogates $~ 850,000$ sites across the genome. Previous studies were performed using Illumina450K BeadChip that covers only $\sim 450,000$ sites. We identified 554 sex associated differentially methylated positions on the autosomes whilst adequately handling the technical bias introduced by normalising with the sex chromosomes and correcting for cross hybridisation of some EPIC probes. Previous work has reported contradicting results, with one group finding that there is higher methylation on autosomes in females $(5,21,60,61)$, another group identifying higher methylation on autosomes in males $(62,63)$ and a third group finding no significant difference in DNAme on autosomes between males and females $(19,64)$. Our results support the former and we found that $70 \%$ of these loci (389 CpGs) showed higher methylation in females compared to males.

The inconsistency seen in the literature is due to the differing normalisation methods applied to DNAme microarray data. Previous research has shown that as the methylation levels of CpG sites on the $X$ chromosome differ largely between males and females, normalisation methods which normalise array data indiscriminately with CpG sites on the autosomes introduce large technical biases for autosomal CpGs (31). Using such normalisation methods, will therefore lead to many autosomal $\mathrm{CpG}$ sites being falsely associated with sex. Our choice of normalisation method greatly reduced technical bias at autosomal CpGs for male and female samples, and, thus, we report a highly robust catalogue of autosomal CpGs differentially methylated between males and females.

We further categorized these 554 saDMPs into two groups, those that were hypermethylated in males $(n=166)$ and those that were hypermethylated in females $(n=389)$. Several saDMPs found to be hypermethylated in females overlapped the transcription start site (TSS) of genes not previously been reported to exhibit sex differences in DNAme including DYRK2, SMAD2 and SHANK2. Interestingly, it has previously been shown that sex hormones can regulate SHANK expression leading to a sex differential expression in SHANK2 (65). Furthermore, this gene has previously been implicated in autism spectrum disorder, a disorder known to exhibit higher prevalence in males rather than females (66) In contrast, the most significant saDMP hypermethylated in males is located in the $\mathrm{CpG}$ island of a gene located on chromosome 21 called GABPA. GABP is a methylation sensitive transcription factor and has previously been 
shown to be a transcriptional activator of Cyp $2 \mathrm{~d}-9$, which is a gene encoding a male specific steroid in mice (67). Sex differences in these regions have previously been identified (21).

Interestingly, as well as DYRK2 and GABPA being the genes annotated to the most significant saDMPs hypermethylated in females and males respectively, they were also the two most significant saDMRs, suggesting these regions could account for important sex biases observed in some diseases. This is further supported by the fact that GABPA has also been heavily associated with early onset of Alzheimers disease, Parkinsons disease, breast cancer and autism (68) and DYRK2 implication in cancer (69). Our KEGG term analysis at those saDMPs hypermethylated in females also implicated many cancer related KEGG terms including thyroid cancer, endometrial cancer, non-small cell lung cancer and more (Figure 2B).

The saDMR harbouring the highest number of CpG sites $(n=58)$ is located on chromosome 6, overlaps SCAND3/ ZBED9 and is hypermethylated in males. Hypermethylation of this gene has been suggested to be implicated in hepatocellular carcinoma which is third leading cause of deaths related to cancer (70). These results collectively support the hypothesis that sex differences in autosomal DNAme may account for some of the sex differences seen in disease prevalence, onset and progression. Moreover, we did identify saDMPs in genes known to exhibit sex differences in DNAme such as CRIPS2, SLC9A2, DDX43 which are involved in spermatogenesis and male fertility $(21,30)$. Specifically, CRIPS2 harboured 9 significant saDMPs, all hypermethylated in females, and is part of a group of proteins called CRISPs which show male biased expression in the male reproductive tract. CRIPS2 plays an important role in spermatogenesis, acrosome reaction and gamete fusion (71). Some of our saDMPs were located in genes known to show sex by age effects, such as PRR4, a gene associated with dry eye syndrome (Perumal et al,. 2016). Despite this, recent research shows that the adult blood DNA methylome is largely affected by sex, but that these methylome sex differences do not change throughout adulthood and so are largely independent from age effects (72, 73).

The Illumina EPIC array has an increased coverage of the genome, including distal regulatory elements (74). It was interesting that the 554 saDMPs were still found to be significantly enriched at $\mathrm{CpG}$ islands and $\mathrm{CpG}$ shores but depleted in open sea regions of the genome (Figure 1E). As the genomic location of DNA methylation normally alters its function, with methylation in $\mathrm{CpG}$ islands normally functioning to serve long term silencing of genes (75) and CpG island shore methylation being strongly related to gene expression (76) this suggests a potential functional role for these saDMPs. To further support these findings, we identified enrichment of these saDMPs at enhancers, 5'UTRs and promoters (Figure 1F). Despite this enrichment at regulatory regions, we found little correlation of these sites alone with differences in gene expression between males and females, suggesting that these saDMPs may not be sufficient alone to predict gene expression. It is worthwhile mentioning that, while some genes associated with these saDMPs displayed statistically significant difference in expression between males and females, the differences in expression were modest.

Instead, these saDMPs are likely to interact with transcription factors and other regulatory features to affect gene expression and chromatin organisation. This potential link was identified in our TF motif analysis, where we found SRY (sex determining region Y) and SOX9 (SRY-box 9) transcription factor motifs also known as the sex determining factors, to be enriched at those saDMPs hypermethylated in females and further identified these as hubs in the TF network. SRY has been found to bind and repress WNT activation 
of ovarian genes, and both SRY and SOX9 transcription factors have been shown to bind the promoter regions of many targets of involved in differentiation of the testis (53). Furthermore, we also found SR1 TFBS enriched in the saDMPs hypermethylated in females, a gene known to interact with SOX9 to increase its own expression to propel differentiation of testis beyond SRY activity (53)

It has previously been reported that $3 \mathrm{D}$ genome organisation can impact sex biased gene expression through direct and indirect effects of cohesion and CTCF looping on enhancer interactions with sex biased genes (77), Recently, it was shown that with rising oestrogen levels, the female brain exhibits sex hormone driven plasticity and that chromatin changes underlie this (78). Interestingly, by annotating our saDMPs to distal genes using chromatin loops, we were able to identify contacts between saDMPs and three genes HIST1H3A, HIST1H4A and HIST1H4B which are core components of nucleosome, thereby responsible for playing a role in chromatin organisation. We further were able to identify interactions between these three genes and a gene harbouring a saDMP found to be hypermethylated in males called CDYL. These results suggest that although we found DNAme to not be predictive of sex differences in gene expression (Figure S5), these saDMPs may interact with other genes, transcription factors and other epigenetic modifications to direct chromatin organisation and regulatory networks.

Lastly, we acknowledge limited overlap with previous studies yet conclude that this is due to our extremely large sample size $(n=1171)$ and improved handling of sex bias introduced by normalising such data with the sex chromosomes. Both of these factors contribute to our ability to detect true positives and obtain a more robust catalogue of true sex associated autosomal CpGs.

\section{AVAILABILITY}

The code to perform this analysis is available on GitHub https://github.com/livygrant97/ASD DNAme

\section{Competing interests}

\section{ACKNOWLEDGEMENT}

The authors acknowledge the use of the High Performance Computing Facility at the University of Essex and would like to thank Stuart Newman for his support.

\section{FUNDING}

OAG is funded by the University of Essex. M.K. was supported by the University of Essex and ESRC (grant RES-596-28-0001). L.S. was supported by Medical Research Council grant K013807. N.R.Z. was supported by the Queen Mary University of London.

Measurement of DNA methylation in Understanding Society: The UK Household Longitudinal Study was funded through an enhancement to Economic and Social Research Council (ESRC) grant ES/N00812X/1.

The analysis was facilitated by access to the Ceres high-performance computing cluster at the University of Essex. 


\section{CONFLICT OF INTEREST}

The authors declare that they have no competing interests.

\section{REFERENCES}

1. Beery,A.K. and Zucker,I. (2011) Sex bias in neuroscience and biomedical research. Neuroscience and Biobehavioral Reviews, 35, 565-572.

2. Credendino,S.C., Neumayer,C. and Cantone,I. (2020) Genetics and Epigenetics of Sex Bias: Insights from Human Cancer and Autoimmunity. Trends in Genetics, 36, 650-663.

3. Hartman,R.J.G., Huisman,S.E. and den Ruijter,H.M. (2018) Sex differences in cardiovascular epigenetics-a systematic review. Biology of Sex Differences, 9, 19.

4. Qin,X., Li,J., Wu,T., Wu,Y., Tang,X., Gao,P., Li,L., Wang,M., Wu,Y., Wang,X., et al. (2019) Overall and sex-specific associations between methylation of the ABCG1 and APOE genes and ischemic stroke or other atherosclerosis-related traits in a sibling study of Chinese population. Clinical Epigenetics, 11, 189.

5. Davegårdh,C., Hall Wedin,E., Broholm,C., Henriksen,T.I., Pedersen,M., Pedersen,B.K., Scheele,C. and Ling,C. (2019) Sex influences DNA methylation and gene expression in human skeletal muscle myoblasts and myotubes. Stem Cell Research and Therapy, 10, 26.

6. Koo,H.K., Morrow,J., Kachroo,P., Tantisira,K., Weiss,S.T., Hersh,C.P., Silverman,E.K. and DeMeo,D.L. (2020) Sex-specific associations with DNA methylation in lung tissue demonstrate smoking interactions. Epigenetics, 10.1080/15592294.2020.1819662.

7. Xia,Y., Dai,R., Wang,K., Jiao,C., Zhang,C., Xu,Y., Li,H., Jing,X., Chen,Y., Jiang,Y., et al. (2021) Sexdifferential DNA methylation and associated regulation networks in human brain implicated in the sexbiased risks of psychiatric disorders. Molecular Psychiatry, 26, 835-848.

8. Smith-Bouvier,D.L., Divekar,A.A., Sasidhar,M., Du,S., Tiwari-Woodruff,S.K., King,J.K., Arnold,A.P., Singh,R.R. and Voskuhl,R.R. (2008) A role for sex chromosome complement in the female bias in autoimmune disease. Journal of Experimental Medicine, 205, 1099-1108.

9. Wijchers,P.J. and Festenstein,R.J. (2011) Epigenetic regulation of autosomal gene expression by sex chromosomes. Trends in Genetics, 27, 132-140.

10. Link,J.C., Chen,X., Arnold,A.P. and Reue,K. (2013) Metabolic impact of sex chromosomes. Adipocyte, 2 , 74-79.

11. Werner,R.J., Schultz,B.M., Huhn,J.M., Jelinek,J., Madzo,J. and Engel,N. (2017) Sex chromosomes drive gene expression and regulatory dimorphisms in mouse embryonic stem cells. Biology of Sex Differences, 8, 1-18.

12. Fish,E.N. (2008) The X-files in immunity: Sex-based differences predispose immune responses. Nature Reviews Immunology, 8, 737-744.

13. Rubtsova,K., Marrack,P. and Rubtsov,A. v. (2015) Sexual dimorphism in autoimmunity. Journal of Clinical Investigation, 125, 2187-2193.

14. BT,M., T,B.-M., S,B., J,B., V,C., C,S. and CT,R. (2016) Large Scale Gene Expression Meta-Analysis Reveals Tissue-Specific, Sex-Biased Gene Expression in Humans. Frontiers in genetics, 7.

15. Lopes-Ramos,C.M., Chen,C.-Y., Kuijjer,M.L., Glass,K., Quackenbush,J. and Demeo Correspondence,D.L. (2020) Sex Differences in Gene Expression and Regulatory Networks across 29 Human Tissues. 10.1016/j.celrep.2020.107795. 
16. A,S. and DJ,W. (2013) Genome-wide analysis of chromatin states reveals distinct mechanisms of sexdependent gene regulation in male and female mouse liver. Molecular and cellular biology, 33, 35943610 .

17. Liu,J., Morgan,M., Hutchison,K. and Calhoun,V.D. (2010) A study of the influence of sex on genome wide methylation. PLOS ONE, 5.

18. Numata,S., Ye,T., Hyde,T.M., Guitart-Navarro,X., Tao,R., Wininger,M., Colantuoni,C., Weinberger,D.R., Kleinman,J.E. and Lipska,B.K. (2012) DNA methylation signatures in development and aging of the human prefrontal cortex. American Journal of Human Genetics, 90, 260-272.

19. Hall,E., Volkov,P., Dayeh,T., Esguerra,J.L. ou S., Salö,S., Eliasson,L., Rönn,T., Bacos,K. and Ling,C. (2014) Sex differences in the genome-wide DNA methylation pattern and impact on gene expression, microRNA levels and insulin secretion in human pancreatic islets. Genome biology, 15, 522.

20. Sun,L., Lin,J., Du,H., Hu,C., Huang,Z., Lv,Z., Zheng,C., Shi,X., Zhang,Y. and Yang,Z. (2014) GenderSpecific DNA Methylome Analysis of a Han Chinese Longevity Population. BioMed Research International, 2014, 1-9.

21. Yousefi,P., Huen,K., Davé,V., Barcellos,L., Eskenazi,B. and Holland,N. (2011) Sex differences in DNA methylation assessed by $450 \mathrm{~K}$ BeadChip in newborns. 10.1186/s12864-015-2034-y.

22. Price,M.E., Cotton,A.M., Lam,L.L., Farré,P., Emberly,E., Brown,C.J., Robinson,W.P. and Kobor,M.S. (2013) Additional annotation enhances potential for biologically-relevant analysis of the Illumina Infinium HumanMethylation450 BeadChip array. Epigenetics and Chromatin, 6, 4.

23. Singmann,P., Shem-Tov,D., Wahl,S., Grallert,H., Fiorito,G., Shin,S.-Y., Schramm,K., Wolf,P., Kunze,S., Baran,Y., et al. (2015) Characterization of whole-genome autosomal differences of DNA methylation between men and women. Epigenetics \& Chromatin, 10.1186/s13072-015-0035-3.

24. Inoshita,M., Numata,S., Tajima,A., Kinoshita,M., Umehara,H., Yamamori,H., Hashimoto,R., Imoto,I. and Ohmori,T. (2015) Sex differences of leukocytes DNA methylation adjusted for estimated cellular proportions. Biology of Sex Differences, 6.

25. Martin,E., Smeester,L., Bommarito,P.A., Grace,M.R., Boggess,K., Kuban,K., Karagas,M.R., Marsit,C.J., O'Shea,T.M. and Fry,R.C. (2017) Sexual epigenetic dimorphism in the human placenta: Implications for susceptibility during the prenatal period. Epigenomics, 9, 267-278.

26. Suderman,M., Simpkin,A., Sharp,G., Gaunt,T., Lyttleton,O., McArdle,W., Ring,S., Davey Smith,G. and Relton,C. (2017) Sex-associated autosomal DNA methylation differences are wide-spread and stable throughout childhood. bioRxiv, 10.1101/118265.

27. AM,I., V,Y., C,K., AM,M., CJ,B. and WP,R. (2021) A cross-cohort analysis of autosomal DNA methylation sex differences in the term placenta. 10.1101/2021.03.08.434471.

28. Blair,J.D. and Price,E.M. (2012) Illuminating Potential Technical Artifacts of DNA-Methylation Array Probes. The American Journal of Human Genetics, 91, 760-762.

29. Chen,Y.A., Lemire,M., Choufani,S., Butcher,D.T., Grafodatskaya,D., Zanke,B.W., Gallinger,S., Hudson,T.J. and Weksberg,R. (2013) Discovery of cross-reactive probes and polymorphic CpGs in the Illumina Infinium HumanMethylation450 microarray. Epigenetics, 8, 203-209.

30. McCarthy,N.S., Melton,P.E., Cadby,G., Yazar,S., Franchina,M., Moses,E.K., Mackey,D.A. and Hewitt,A.W. (2014) Meta-analysis of human methylation data for evidence of sex-specific autosomal patterns. BMC Genomics, 15, 981. 
31. Wang,Y., Hannon,E., Grant,O.A., Gorrie-Stone,T.J., Kumari,M., Mill,J., Zhai,X., McDonald-Maier,K.D. and Schalkwyk,L.C. (2020) DNA methylation-based sex classifier to predict sex and identify sex chromosome aneuploidy. bioRxiv, 10.1101/2020.10.19.345090.

32. G,K. (2015) Understanding Society—UK Household Longitudinal Study: Wave 1-5, User Manual. Colchester, United Kingdom.

33. Benzeval, Michaela, Davillas, Apostolos, Kumari, Meena, Lynn,P. (2014) No Title. Understanding Society:UK Household Longitudinal Study: Biomarker User Guide and Glossary. Colchester: University of Essex.

34. Hughes,A., Smart,M., Gorrie-Stone,T., Hannon,E., Mill,J., Bao,Y., Burrage,J., Schalkwyk,L. and Kumari,M. (2018) Socioeconomic Position and DNA Methylation Age Acceleration Across the Life Course. American Journal of Epidemiology, 187, 2346-2354.

35. Gorrie-Stone,T.J., Smart,M.C., Saffari,A., Malki,K., Hannon,E., Burrage,J., Mill,J., Kumari,M. and Schalkwyk,L.C. (2019) Bigmelon: Tools for analysing large DNA methylation datasets. Bioinformatics, 35, 981-986.

36. R.,P. A data-driven approach to preprocessing Illumina $450 \mathrm{~K}$ methylation array data. BMC Genomics, 14, 293.

37. Pidsley,R., Y Wong,C.C., Volta,M., Lunnon,K., Mill,J. and Schalkwyk,L.C. (2013) A data-driven approach to preprocessing Illumina 450K methylation array data. BMC Genomics, 14, 293.

38. Morris,T.J., Butcher,L.M., Feber,A., Teschendorff,A.E., Chakravarthy,A.R., Wojdacz,T.K. and Beck,S. (2014) ChAMP: 450k Chip Analysis Methylation Pipeline. Bioinformatics, 30, 428-430.

39. Aryee,M.J., Jaffe,A.E., Corrada-Bravo,H., Ladd-Acosta,C., Feinberg,A.P., Hansen,K.D. and Irizarry,R.A. (2014) Minfi: A flexible and comprehensive Bioconductor package for the analysis of Infinium DNA methylation microarrays. Bioinformatics, 30, 1363-1369.

40. B,P., J,M. and A,O. (2016) missMethyl: an R package for analyzing data from Illumina's HumanMethylation450 platform. Bioinformatics (Oxford, England), 32, 286-288.

41. Yu,G., Wang,L.G., Han,Y. and He,Q.Y. (2012) ClusterProfiler: An R package for comparing biological themes among gene clusters. OMICS A Journal of Integrative Biology, 16, 284-287.

42. Stojnic R,D.D. (2020) PWMEnrich: PWM enrichment analysis. R package verion 4.26.0.

43. Shannon P and Richards M (2021) MotifDb: An Annotated Collection of Protein-DNA Binding Sequence Motifs. $R$ package version 1.34.0.

44. Love,M.I., Huber,W. and Anders,S. (2014) Moderated estimation of fold change and dispersion for RNAseq data with DESeq2. Genome Biology, 15, 550.

45. Durand,N.C., Shamim,M.S., Machol,I., Rao,S.S.P., Huntley,M.H., Lander,E.S. and Aiden,E.L. (2016) Juicer Provides a One-Click System for Analyzing Loop-Resolution Hi-C Experiments. Cell Systems, 3 , 95-98.

46. Li,H. and Durbin,R. (2010) Fast and accurate long-read alignment with Burrows-Wheeler transform. Bioinformatics, 26, 589-595.

47. Shannon,P., Markiel,A., Ozier,O., Baliga,N.S., Wang,J.T., Ramage,D., Amin,N., Schwikowski,B. and Ideker,T. (2003) Cytoscape: A software Environment for integrated models of biomolecular interaction networks. Genome Research, 13, 2498-2504.

48. Chathoth,K.T. and Zabet,N.R. (2019) Chromatin architecture reorganization during neuronal cell differentiation in Drosophila genome. Genome Research, 29, 613-625. 
49. Nasser,J., Bergman,D.T., Fulco,C.P., Guckelberger,P., Doughty,B.R., Patwardhan,T.A., Jones,T.R., Nguyen,T.H., Ulirsch,J.C., Lekschas,F., et al. (2021) Genome-wide enhancer maps link risk variants to disease genes. Nature 2021 593:7858, 593, 238-243.

50. Xia,X., Zhou,X., Quan,Y., Hu,Y., Xing,F., Li,Z., Xu,B., Xu,C. and Zhang,A. (2019) Germline deletion of Cdyl causes teratozoospermia and progressive infertility in male mice. Cell Death \& Disease 2019 10:3, 10, 1-13.

51. Gómez-Abellán,P., Madrid,J.A., Luján,J.A., Frutos,M.D., González,R., Martínez-Augustín,O., de Medina,F.S., Ordovás,J.M. and Garaulet,M. (2012) Sexual dimorphism in clock genes expression in human adipose tissue. Obesity Surgery, 22, 105-112.

52. StelzI,U., Worm,U., Lalowski,M., Haenig,C., Brembeck,F.H., Goehler,H., Stroedicke,M., Zenkner,M., Schoenherr,A., Koeppen,S., et al. (2005) A human protein-protein interaction network: A resource for annotating the proteome. Cell, 122, 957-968.

53. Li,Y., Zheng,M. and Lau,Y.F.C. (2014) The sex-determining factors SRY and SOX9 regulate similar target genes and promote testis cord formation during testicular differentiation. Cell Reports, 8, 723733.

54. Fatima,L.A., Campello,R.S., Barreto-Andrade,J.N., Passarelli,M., Santos,R.S., Clegg,D.J. and Machado,U.F. (2019) Estradiol stimulates adipogenesis and SIc2a4/GLUT4 expression via ESR1mediated activation of CEBPA. Molecular and Cellular Endocrinology, 498, 110447.

55. Alogayil,N., Bauermeister,K., Hector Galvez,J., Venkatesh,V.S., Kim-Wee Zhuang,Q., Chang,M.L., Davey,R.A., Zajac,J.D., Ida,K., Kamiya,A., et al. (123AD) Distinct roles of androgen receptor, estrogen receptor alpha, and BCL6 in the establishment of sex-biased DNA methylation in mouse liver. Scientific Reports /, 11, 13766.

56. Al-Moghrabi,N., Nofel,A., Al-Yousef,N., Madkhali,S., bin Amer,S.M., Alaiya,A., Shinwari,Z., AlTweigeri,T., Karakas,B., Tulbah,A., et al. (2014) The molecular significance of methylated BRCA1 promoter in white blood cells of cancer-free females. BMC Cancer, 14, 1-12.

57. Wiseman,F.K., Al-Janabi,T., Hardy,J., Karmiloff-Smith,A., Nizetic,D., Tybulewicz,V.L.J., Fisher,E.M.C. and Strydom,A. (2015) A genetic cause of Alzheimer disease: Mechanistic insights from Down syndrome. Nature Reviews Neuroscience, 16, 564-574.

58. Li,Y., Xu,A., Jia,S. and Huang,J. (2019) Recent advances in the molecular mechanism of sex disparity in hepatocellular carcinoma (review). Oncology Letters, 17, 4222-4228.

59. Somineni,H.K., Venkateswaran,S., Kilaru,V., Marigorta,U.M., Mo,A., Okou,D.T., Kellermayer,R., Mondal,K., Cobb,D., Walters,T.D., et al. (2019) Blood-Derived DNA Methylation Signatures of Crohn's Disease and Severity of Intestinal Inflammation. Gastroenterology, 156, 2254-2265.e3.

60. Singmann,P., Shem-Tov,D., Wahl,S., Grallert,H., Fiorito,G., Shin,S.Y., Schramm,K., Wolf,P., Kunze,S., Baran,Y., et al. (2015) Characterization of whole-genome autosomal differences of DNA methylation between men and women. Epigenetics and Chromatin, 8.

61. Xu,H., Wang,F., Liu,Y., Yu,Y., Gelernter,J. and Zhang,H. (2014) Sex-biased methylome and transcriptome in human prefrontal cortex. Human Molecular Genetics, 23, 1260-1270.

62. Zhang,F.F., Cardarelli,R., Carroll,J., Fulda,K.G., Kaur,M., Gonzalez,K., Vishwanatha,J.K., Santella,R.M. and Morabia,A. (2011) Significant differences in global genomic DNA methylation by gender and race/ethnicity in peripheral blood. Epigenetics, 6, 623-629.

63. García-Calzón,S., Perfilyev,A., de Mello,V.D., Pihlajamäki,J. and Ling,C. (2018) Sex differences in the methylome and transcriptome of the human liver and circulating HDL-cholesterol levels. Journal of Clinical Endocrinology and Metabolism, 103, 4395-4408. 
64. Eckhardt,F., Lewin,J., Cortese,R., Rakyan,V.K., Attwood,J., Burger,M., Burton,J., Cox,T. v., Davies,R., Down,T.A., et al. (2006) DNA methylation profiling of human chromosomes 6, 20 and 22. Nature Genetics, 38, 1378-1385.

65. Berkel,S., Eltokhi,A., Fröhlich,H., Porras-Gonzalez,D., Rafiullah,R., Sprengel,R. and Rappold,G.A. (2018) Sex Hormones Regulate SHANK Expression. Frontiers in Molecular Neuroscience, 11, 337.

66. Mottron,L., Duret,P., Mueller,S., Moore,R.D., Forgeot D'Arc,B., Jacquemont,S. and Xiong,L. (2015) Sex differences in brain plasticity: A new hypothesis for sex ratio bias in autism Understanding the links between sex/gender and autism Dr Meng-Chuan Lai. Molecular Autism, 6, 1-19.

67. N,Y., R,K., R,M., T,S. and M,N. (1995) A DNA methylation site in the male-specific P450 (Cyp 2d-9) promoter and binding of the heteromeric transcription factor GABP. Molecular and cellular biology, 15, $5355-5362$.

68. Caspermeyer,J. (2016) Scientists Explore a Master Genetic Switch That Plays a Key Role in Energy Metabolism and Human Brain Evolution and Function. Molecular Biology and Evolution, 33, 1376.21376.

69. Tandon,V., de La Vega,L. and Banerjee,S. (2021) Emerging roles of DYRK2 in cancer. Journal of Biological Chemistry, 296.

70. Xu,F., Zhang,L., Xu,Y., Song,D., He,W., Ji,X. and Shao,J. (2020) Hypermethylation of scand3 and myo1g gene are potential diagnostic biomarkers for hepatocellular carcinoma. Cancers, 12, 1-15.

71. Lim,S., Kierzek,M., O'Connor,A.E., Brenker,C., Merriner,D.J., Okuda,H., Volpert,M., Gaikwad,A., Bianco,D., Potter,D., et al. (2019) CRISP2 is a regulator of multiple aspects of sperm function and male fertility. Endocrinology, 160, 915-924.

72. Bergstedt,J., Ait,S., Azzou,K., Tsuo,K., Jaquaniello,A., Urrutia,A., Rotival,M., Lin,D.T.S., Macisaac,J.L., Kobor,M.S., et al. Factors Driving DNA Methylation Variation in Human Blood. 10.1101/2021.06.23.449602.

73. Gatev,E., Inkster,A.M., Negri,G.L., Konwar,C., Lussier,A.A., Skakkebaek,A., Sokolowski,M.B., Gravholt,C.H., Dunn,E.C., Kobor,M.S., et al. (2021) Autosomal sex-associated co-methylated regions predict biological sex from DNA methylation. Nucleic Acids Research, 10.1093/NAR/GKAB682.

74. Pidsley,R., Zotenko,E., Peters,T.J., Lawrence,M.G., Risbridger,G.P., Molloy,P., van Djik,S., Muhlhausler,B., Stirzaker,C. and Clark,S.J. (2016) Critical evaluation of the Illumina MethylationEPIC BeadChip microarray for whole-genome DNA methylation profiling. Genome Biology, 17, 1-17.

75. Jones,P.A. (2012) Functions of DNA methylation: islands, start sites, gene bodies and beyond. $10.1038 /$ nrg3230.

76. Irizarry,R.A., Ladd-Acosta,C., Wen,B., Wu,Z., Montano,C., Onyango,P., Cui,H., Gabo,K., Rongione,M., Webster,M., et al. (2009) The human colon cancer methylome shows similar hypo- and hypermethylation at conserved tissue-specific CpG island shores. Nature Genetics, 41, 178-186.

77. Matthews,B.J. and Waxman,D.J. (2020) Impact of 3D genome organization, guided by cohesin and CTCF looping, on sex-biased chromatin interactions and gene expression in mouse liver. Epigenetics \& Chromatin 2020 13:1, 13, 1-25.

78. Rocks,D., Shukla,M., Finnemann,S.C., Kalluchi,A., Jordan Rowley,M. and Kundakovic,M. (2021) Sexspecific multi-level 3D genome dynamics in the mouse brain. bioRxiv, 10.1101/2021.05.03.442383. 


\section{TABLE AND FIGURES LEGENDS}

Table 1. Enriched GO terms among the $14,653 \mathrm{CpGs}$ identified to be significantly associated with sex. $\mathrm{N}^{1}$ indicates the number of genes in the GO term. $D E^{2}$ refers to the number of genes annotated to the sex associated DMPs which are differentially methylated. P.DE ${ }^{3}$ indicates the $P$ value for over representation of the GO term in this data set. $\mathrm{FDR}^{4}$ indicates the false discovery rate (using the Benjamini and Hochberg method).

Figure 1: Location and characterisation of saDMPs. (A) Manhattan plot for EWAS analysis of sex. CpG sites which met a threshold of FDR $<0.05$ and had an average beta change of $>0.05$ were considered significant and are represented by darker colours. (B) Volcano plot for saDMPs. CpGs which are not significant are represented in grey, saDMPs hypermethylated in males are in orange and saDMPs hypermethylated in females in blue. (C) Principal component analysis of beta values at the significant saDMPs. Male samples are indicated in orange while female samples are indicated in blue. (D) Number of saDMPs harboured by individual genes. (E) Top panel shows the annotation of all saDMPs $(n=544)$, saDMPs hypermethylated in females ( $n=382)$ and saDMPs hypermethylated in males $(n=162)$ relative to $\mathrm{CpG}$ island regions compared to the autosomal background. Bottom panel shows the $\log _{2}$ (obs/exp) annotations based on the autosomal background of the different annotations. (F) Top panel shows the overlap of all saDMPs ( $n=544)$, saDMPs hypermethylated in females ( $n=382)$ and saDMPs hypermethylated in males $(n=162)$ with genomic features compared to the autosomal background. Bottom panel shows the $\log _{2}$ (obs/exp) annotations based on the autosomal background of the different annotations.

Figure 2: Transcription factor motif enrichment analysis. (A) Overlap of enriched TF motifs for saDMPs hypermethylated in females (blue) and saDMPs hypermethylated in males (orange). saDMPs found to be hypermethylated in females were enriched in TF binding motifs including SOX9 and SRY. (B) KEGG analyses for the significantly enriched TF motifs at saDMPs hypermethylated in females. (C-D) Subnetworks of the top 30 enriched TF motifs at saDMPs hypermethylated in males (C) and females (D). Node colour represents the degree of connectivity. The scale from red to yellow represents the top 30 enriched TF motif rank from 1-30, with red indicating highest degree and yellow indicating lowest degree.

Figure 3. Plots of sex associated differentially methylated regions (saDMR). We plotted regions: (A) GABPA and ATP5J, (B) DYRK2, (C) SMAD2 and (D) PRKXP1. Yellow boxes represent appropriately labelled genes, green boxes represent the genomic region which the differentially methylated region spans. The scatterplots represent the methylation values for males (orange) and females (blue) at $\mathrm{CpG}$ sites located within the differentially methylated region.

Figure S1: (A) QQ plot and lambda values for the distribution of the adjusted $p$ values against the null distribution for EWAS of sex in the understanding society cohort. Genomic inflation lambda score is indicated in the QQ plot to indicate statistical inflation of p-values. (B) Boxplots of estimated whole blood cell type proportions for males (orange) and females (blue), estimated using the estimateCellCounts function from 
bigmelon. We performed a Mann-Whitney U test (p-value: n.s. $\geq 0.05,{ }^{*} p$-value $<0.05,{ }^{* *}<0.01$ and ${ }^{* * *}<$ 0.001).

Figure S2: (A) Integrated genomics viewer track of chromatin loop on chromosome 6 showing two saDMPs hypermethylated in males contacting $\mathrm{H} 1 / \mathrm{H} 4 / \mathrm{H} 3 / \mathrm{H} 2 \mathrm{~V} / \mathrm{H} 2 \mathrm{~A}$. (B) Integrated genomics viewer track of chromatin loop on chromosome 1 showing an saDMP hypermethylated in females contacting the ODF2L gene. Blue lines represent the chromatin loops, with black lines showing the loop anchors. Orange vertical lines represent the saDMPs hypermethylated in males and blue vertical lines represent the saDMPs hypermethylated in females. Purple annotations represent genes. (C-D) Subnetworks of the top 50 genes annotated to saDMPs hypermethylated in males (C) and females (D). Node colour represents the degree of connectivity. The scale from red to yellow represents the top 50 enriched TF motif rank from 1-50, with red indicating highest degree and yellow indicating lowest degree.

Figure S3: GO terms overrepresented for the significantly enriched TF motifs at saDMPs hypermethylated in males $(A)$ and females $(B)$.

Figure S4: (A-B) Network visualisation of protein-protein interactions for all TF motifs enriched at saDMPs hypermethylated in males (A) and females (B). Grey circles represent individual TFs located on autosomes, while purple circles represent TFs encoded on the $\mathrm{X}$ chromosome.

Figure S5: Volcano plot showing differential gene expression between males and females. We considered the case of: (A) genes annotated to the saDMPs, (B) sex chromosome linked genes and (C) autosomal genes. Points coloured in grey represent non differentially expressed genes. Green points represent genes which had a $\log _{2}$ Fold Change value greater than 1 . Blue points represent genes which met the $p$ value threshold $(<0.05)$. Points coloured in red represent genes which showed differential expression between males and females ( $p$ value $<0.05 \& \log _{2} F C>1$ ).

\section{ADDITIONAL FILES}

Additional file 1: Significant sex associated autosomal DMPs. Illumina Manifest annotations for all 554 significant CpG sites associated with sex on the autosomes.

Additional file 2: Significant sex associated autosomal DMRs. Test results for all significant DMR's associated with sex on the autosomes ordered by FDR value.

Additional file 3: Enrichment statistics for the TF motifs enriched at saDMPs hypermethylated in females.

Additional file 4: Enrichment statistics for the TF motifs enriched at saDMPs hypermethylated in males.

Additional file 5: Functional enrichment for subnetworks of TF motifs enriched at saDMPs hypermethylated in females.

Additional file 6: Functional enrichment for subnetworks of TF motifs enriched at saDMPs hypermethylated in males. 

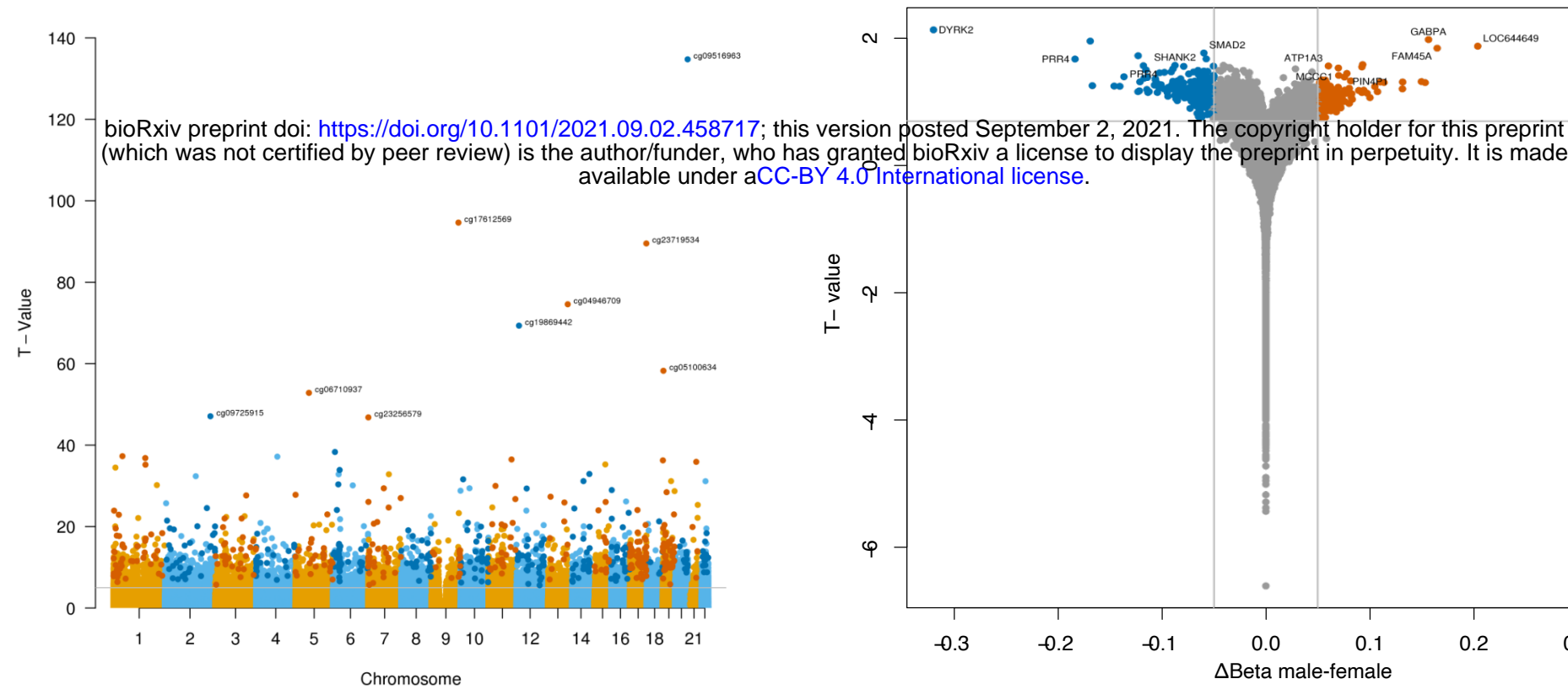

C

PCA
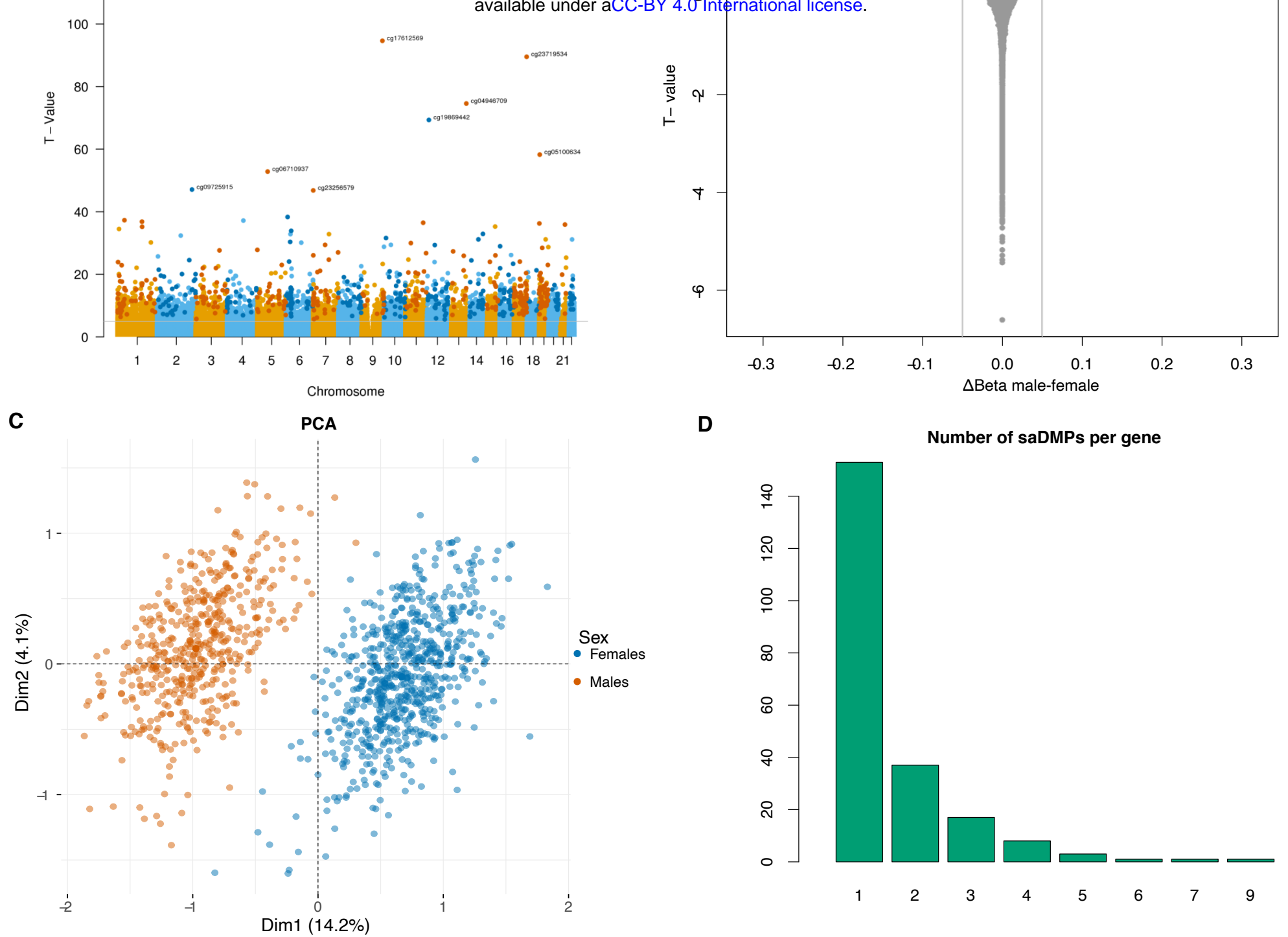

D

Number of saDMPs per gene

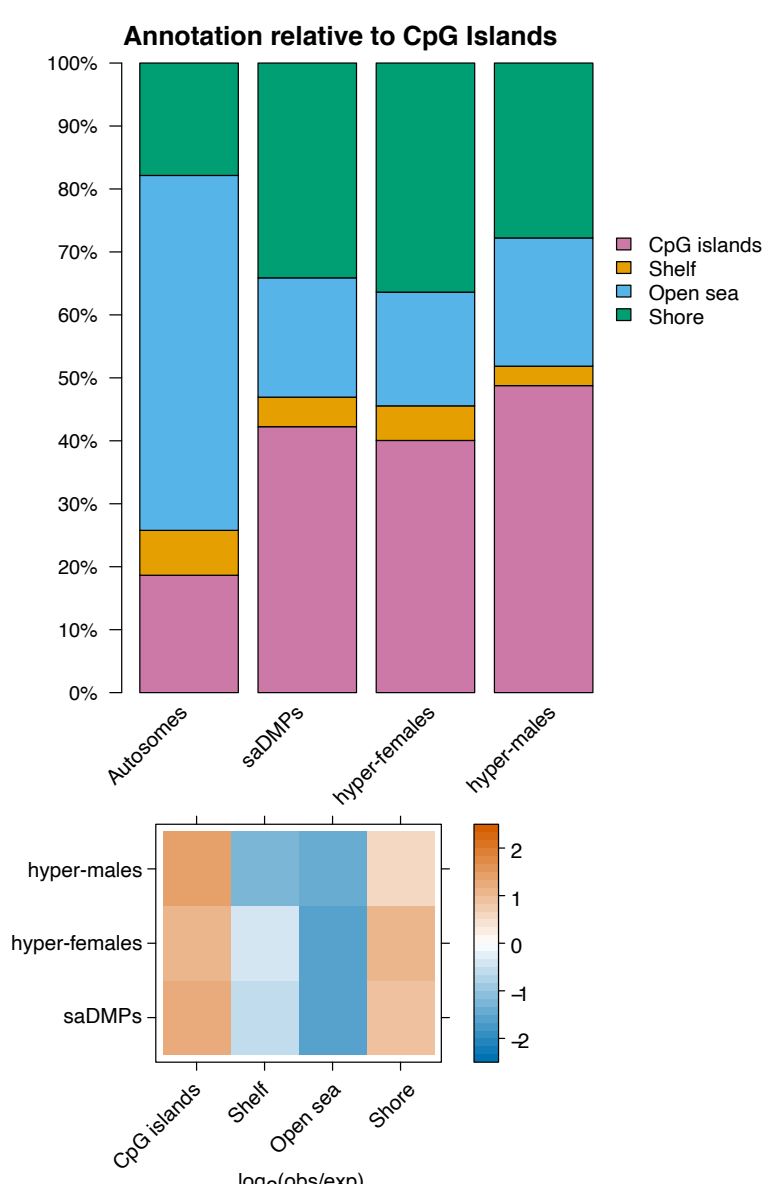

F
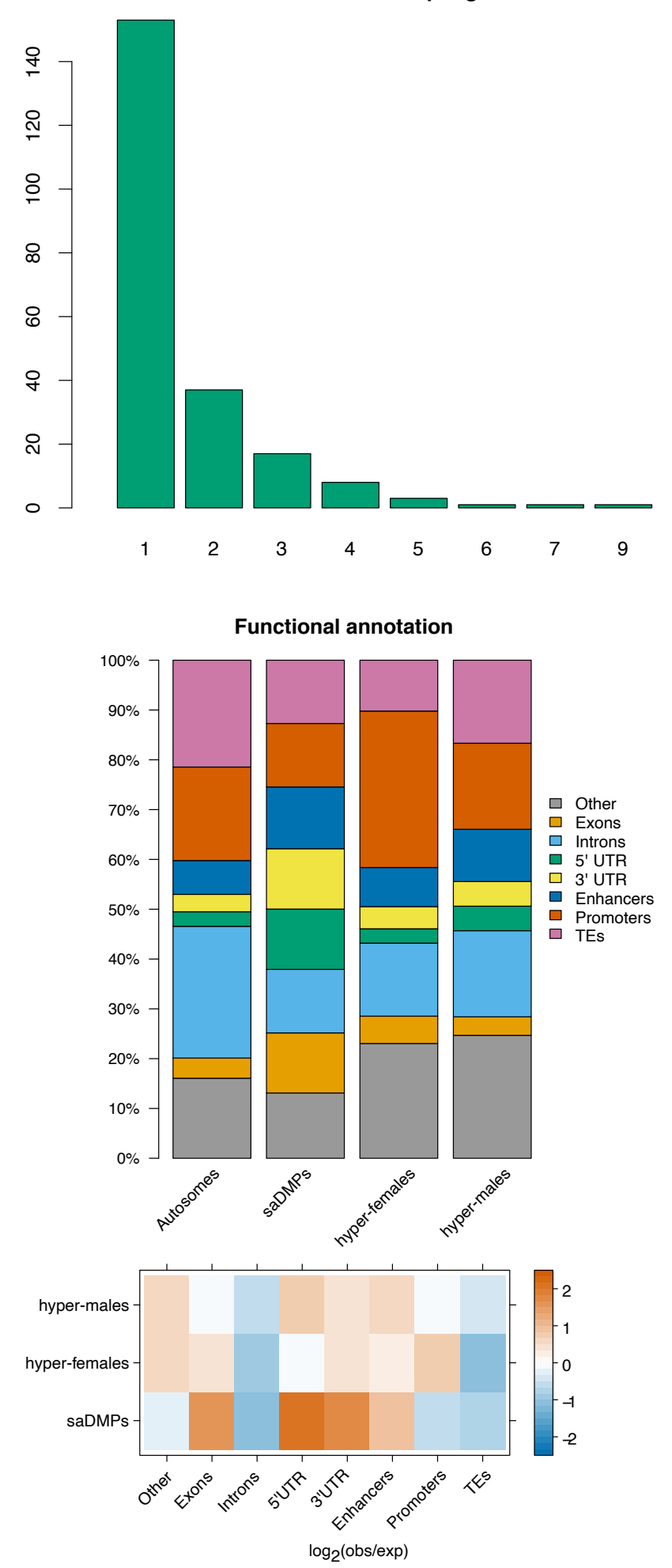

$\log _{2}$ (obs/exp) 

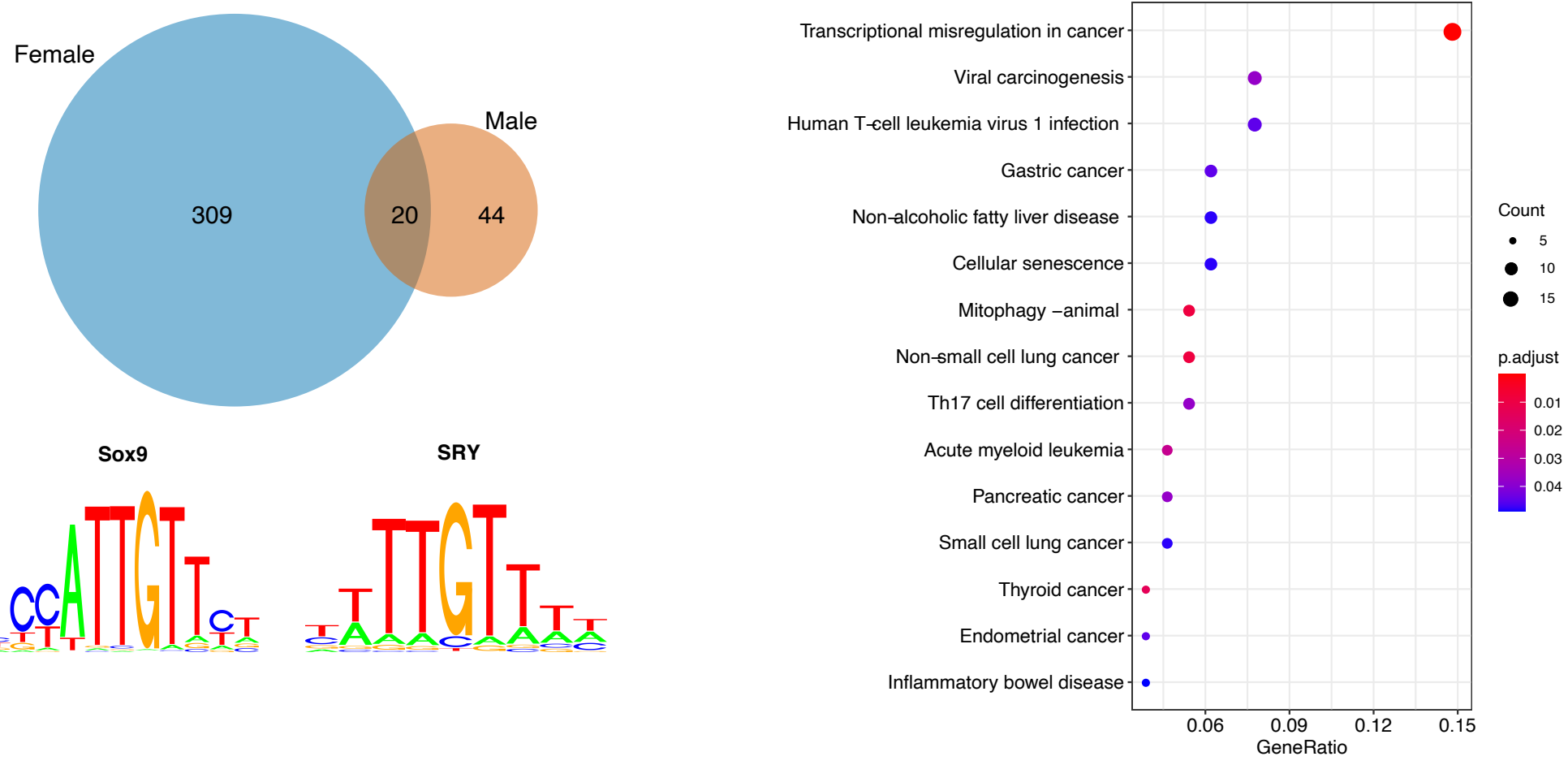

C

Top TF motifs at saDMPs hypermethylated in males

D

Top TF motifs at saDMPs hypermethylated in females

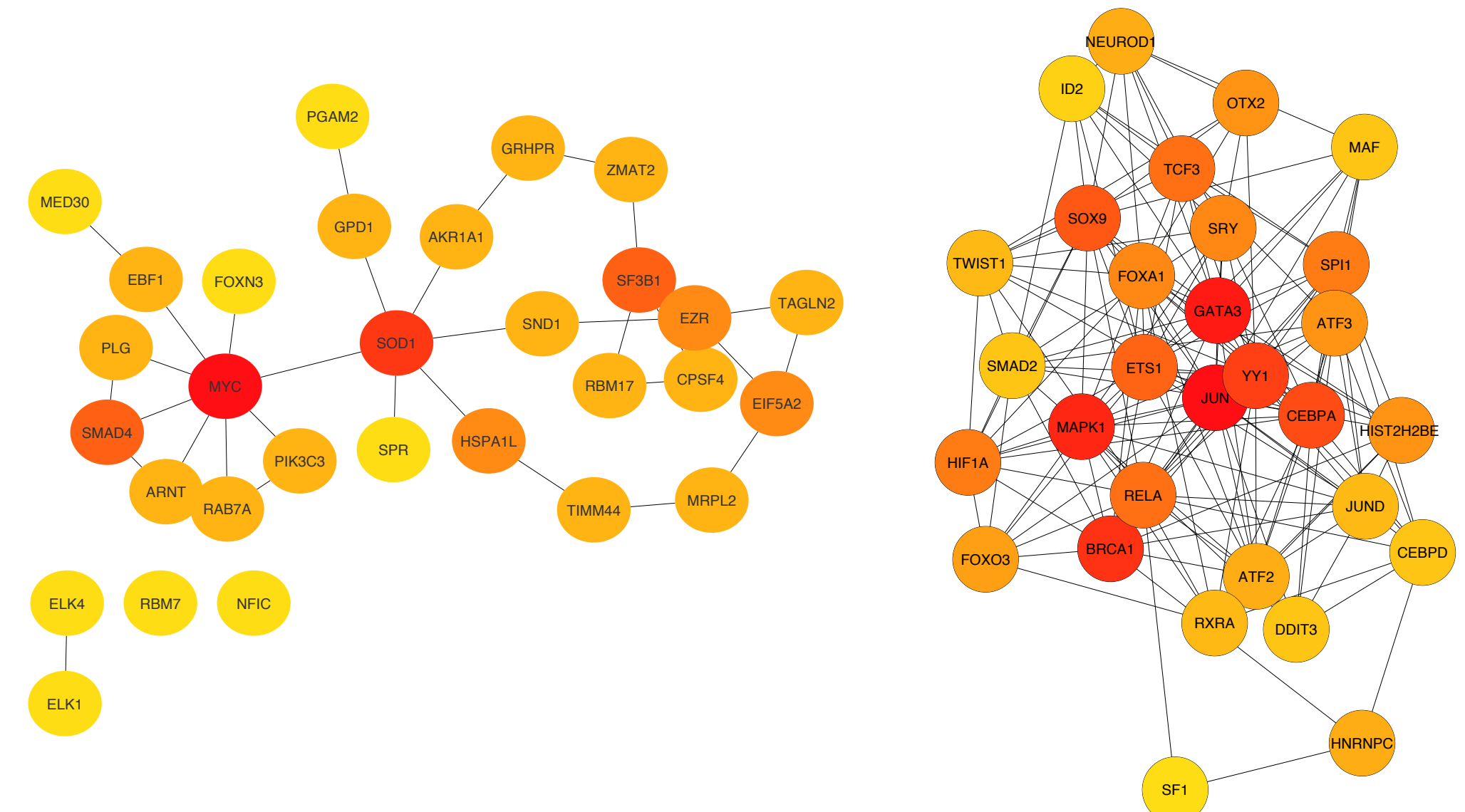



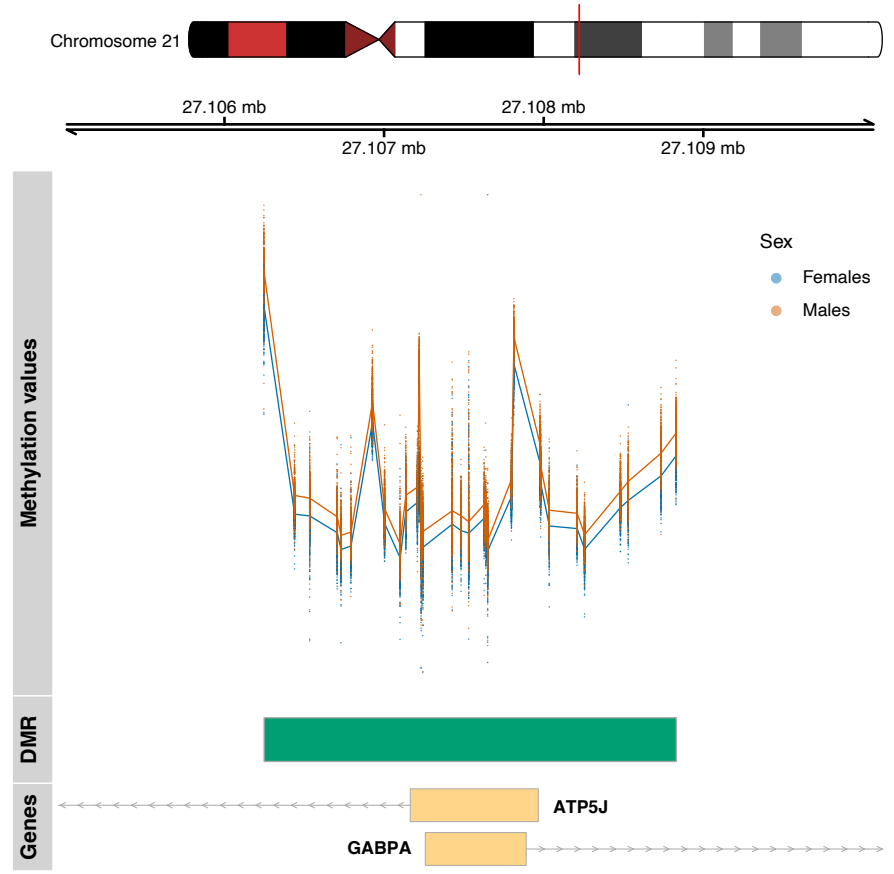

C
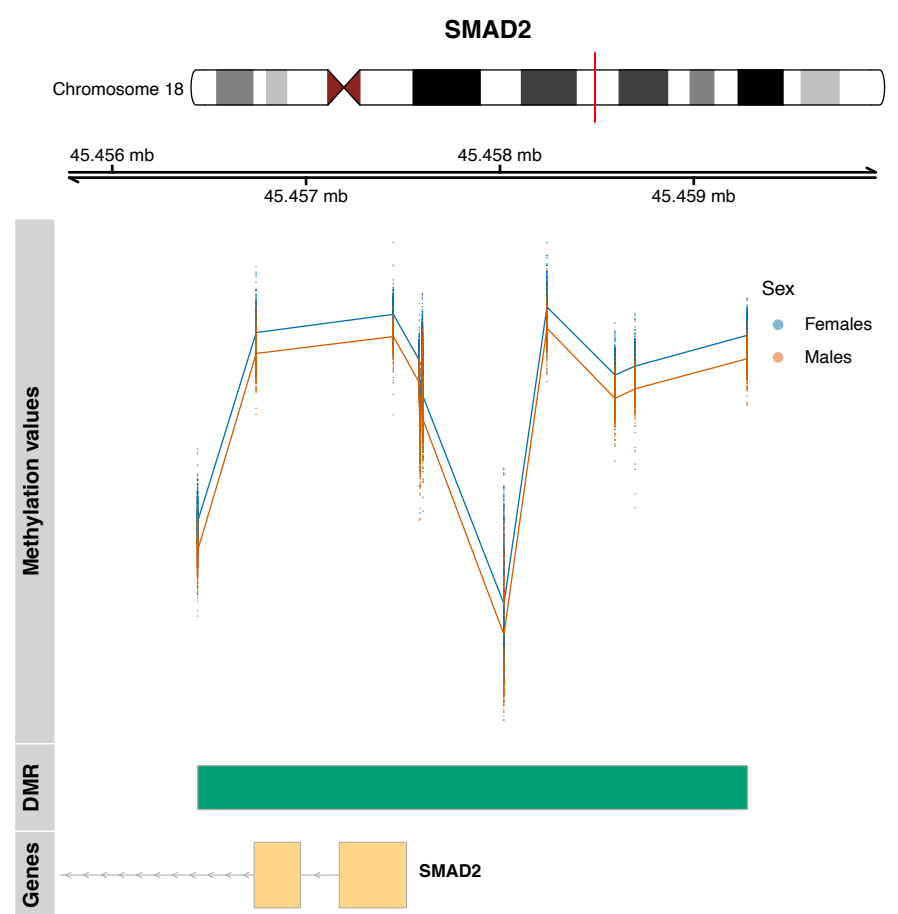

Chromosome ${ }_{12}$ पIIT $68.041 \mathrm{mb}$ $68.043 \mathrm{mb}$ $68.042 \mathrm{mb}$ $68.044 \mathrm{mb}$
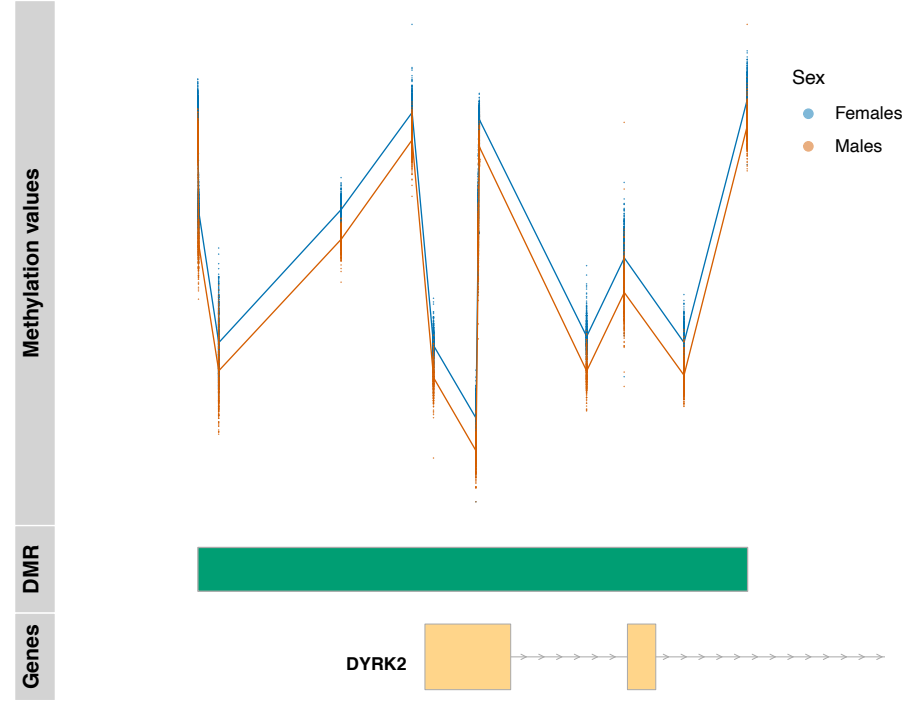

高

¿ัँ

DYRK2

D Chromosome 15 PRKXP1
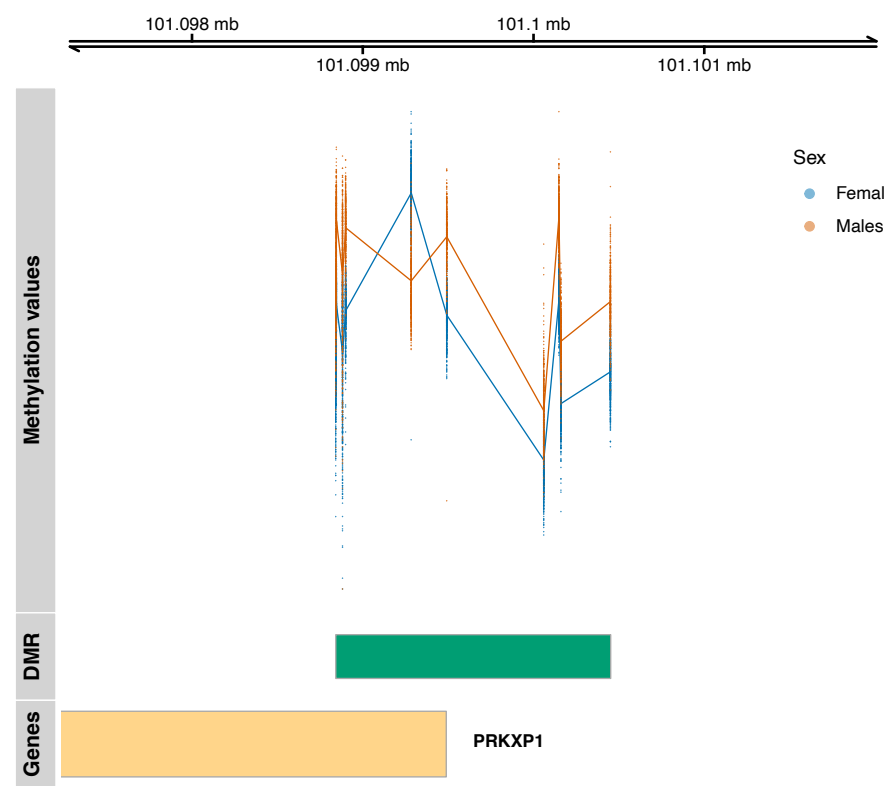

- Females - Males 


\begin{tabular}{|c|c|c|c|c|c|c|}
\hline GO term & Term & Ont & $\mathbf{N}^{1}$ & $\mathrm{DE}^{2}$ & P.DE $^{3}$ & $\mathrm{FDR}^{4}$ \\
\hline GO:0048856 & $\begin{array}{l}\text { anatomical structure } \\
\text { development }\end{array}$ & BP & 5493 & 1820 & $1.27 \mathrm{E}-12$ & 2.53E-08 \\
\hline GO:0044459 & plasma membrane part & CC & 2610 & 934 & $2.24 \mathrm{E}-12$ & $2.53 \mathrm{E}-08$ \\
\hline GO:0071944 & cell periphery & CC & 5023 & 1616 & $4.32 \mathrm{E}-12$ & $3.26 \mathrm{E}-08$ \\
\hline GO:0005886 & plasma membrane & CC & 4919 & 1580 & $6.86 \mathrm{E}-12$ & $3.88 \mathrm{E}-08$ \\
\hline GO:0007275 & $\begin{array}{l}\text { multicellular organism } \\
\text { development }\end{array}$ & BP & 5048 & 1677 & $1.10 \mathrm{E}-11$ & 4.19E-08 \\
\hline GO:0048468 & cell development & $\mathrm{BP}$ & 1999 & 768 & $1.14 \mathrm{E}-11$ & 4.19E-08 \\
\hline GO:0032501 & multicellular organismal process & $\mathrm{BP}$ & 7000 & 2160 & $1.30 \mathrm{E}-11$ & 4.19E-08 \\
\hline GO:0032502 & developmental process & $\mathrm{BP}$ & 5848 & 1902 & $1.49 \mathrm{E}-10$ & 4.21E-07 \\
\hline GO:0009653 & $\begin{array}{l}\text { anatomical structure } \\
\text { morphogenesis }\end{array}$ & BP & 2493 & 910 & 3.23E-10 & 8.11E-07 \\
\hline GO:0048731 & system development & $\mathrm{BP}$ & 4537 & 1506 & $1.22 \mathrm{E}-09$ & 2.64E-06 \\
\hline GO:0030154 & cell differentiation & $\mathrm{BP}$ & 3882 & 1294 & $1.28 \mathrm{E}-09$ & 2.64E-06 \\
\hline GO:0043269 & regulation of ion transport & $\mathrm{BP}$ & 628 & 255 & 2.98E-09 & $5.61 \mathrm{E}-06$ \\
\hline GO:0006811 & ion transport & $\mathrm{BP}$ & 1543 & 550 & $6.78 \mathrm{E}-09$ & $1.18 \mathrm{E}-05$ \\
\hline GO:0048869 & cellular developmental process & $\mathrm{BP}$ & 4054 & 1339 & $9.49 E-09$ & $1.53 \mathrm{E}-05$ \\
\hline GO:0009887 & animal organ morphogenesis & $\mathrm{BP}$ & 957 & 384 & $1.23 \mathrm{E}-08$ & $1.86 \mathrm{E}-05$ \\
\hline GO:0016020 & membrane & CC & 8539 & 2569 & $1.74 \mathrm{E}-08$ & $2.46 \mathrm{E}-05$ \\
\hline GO:0098916 & $\begin{array}{l}\text { anterograde trans-synaptic } \\
\text { signaling }\end{array}$ & $\mathrm{BP}$ & 663 & 276 & 2.50E-08 & 3.14E-05 \\
\hline GO:0007268 & chemical synaptic transmission & $\mathrm{BP}$ & 663 & 276 & $2.50 \mathrm{E}-08$ & 3.14E-05 \\
\hline GO:0044425 & membrane part & CC & 6238 & 1866 & $4.20 \mathrm{E}-08$ & $5.00 \mathrm{E}-05$ \\
\hline GO:0099537 & trans-synaptic signaling & $\mathrm{BP}$ & 670 & 277 & $5.96 \mathrm{E}-08$ & $6.73 \mathrm{E}-05$ \\
\hline
\end{tabular}




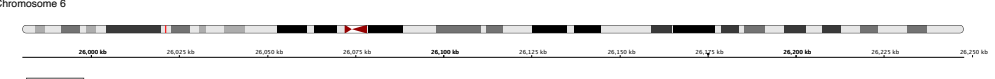

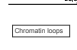

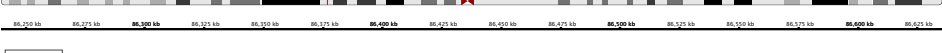

Chmoath noos

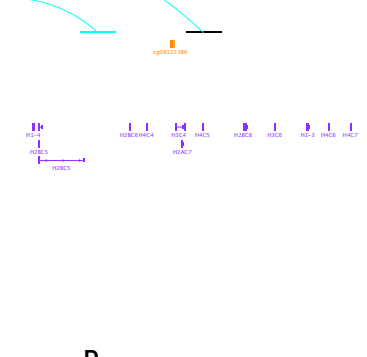

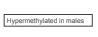

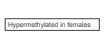

tenst

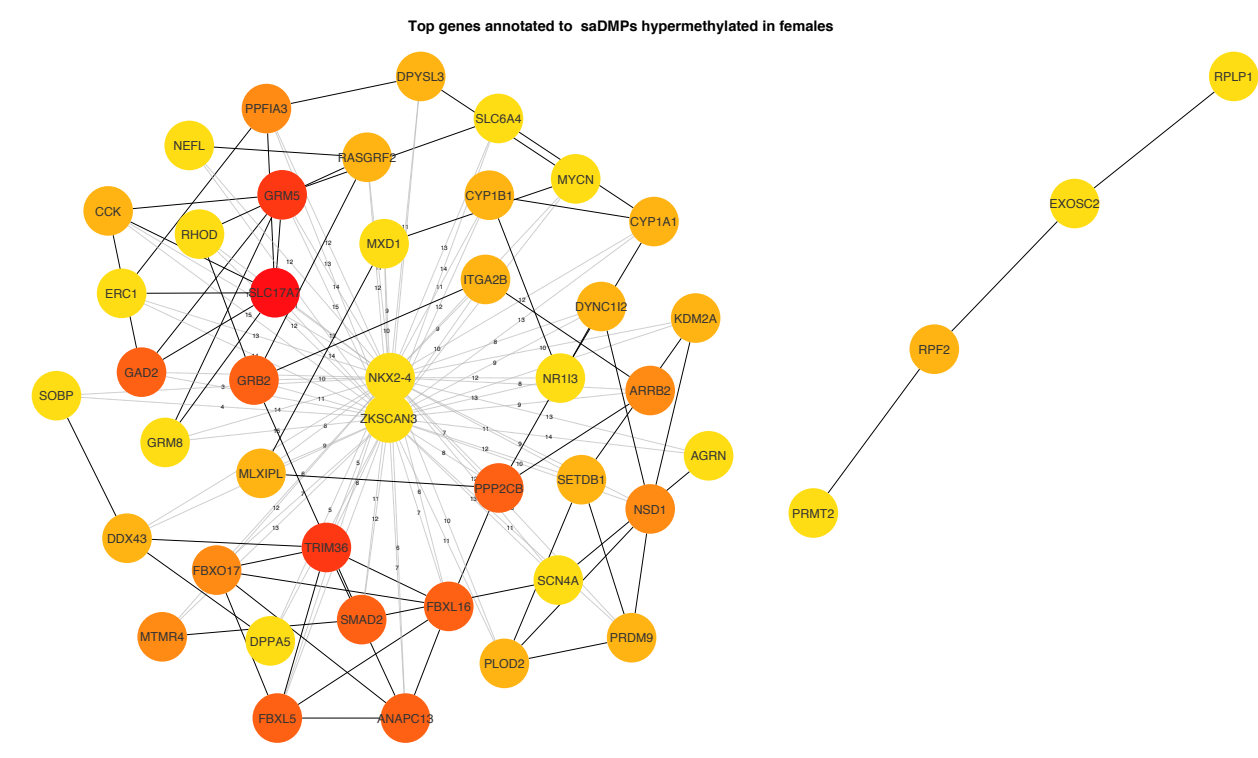

KATNBL1

SPESP1

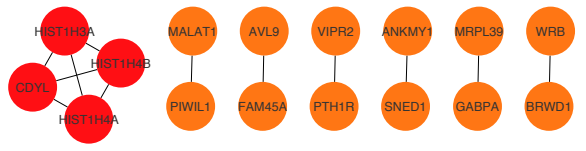

ATP10D KIF26A BAHCC1 PCDHGA4 GRM2 ATP1A3 PRSS50 CNTD2 SLC39A4 PRR15 KIF25

$\begin{array}{lllllll}\text { FRMD8 HGNC:9979 COL25A1 KCNN8 BNIP3 PER3 MUC4 } & \text { OLFM1 NRD1 }\end{array}$ UPF1 MYT1L SLC6A18 PRR25 ZMIZ1 SPATA13 ATP8B3 ADAMTS2 MCCC1 B3GNT1 SMIM24 ISOC2 ZNF718 FOX12

\section{D}


transcription factor activity, RNA polymerase II proximal promoter sequence-specific DNA binding -

DNA-binding transcription activator activity, RNA polymerase II-specific

proximal promoter DNA-binding transcription activator activity, RNA polymerase II-specific

DNA-binding transcription repressor activity, RNA polymerase II-specific

oxidoreductase activity, acting on the $\mathrm{CH}-\mathrm{OH}$ group of donors, NAD or NADP as acceptor

oxidoreductase activity, acting on $\mathrm{CH}-\mathrm{OH}$ group of donors

NADP binding

NAD binding

S100 protein binding

0.05

GeneRatio

Count

- 2

- 6

- 8

padjust

\section{GO enrichment analysis for TF motifs enriched at saDMPs hypermethylated in females}

DNA-binding transcription activator activity, RNA polymerase II-specific proximal promoter DNA pinding transcription activator activity, RNA polymerase II-specific DNA-binding transcription activator activity, RNA polymerase $\|$-specific
Depressor activity, RNA polymerase $\|$-specific

DNA-binding transcription repressor activity, RNA polymerase II-specific
transcription coactivator activity mRNA binding
mans

transcription factor activity, RNA polymerase II distal enhancer sequence-specific binding

transcription corepressor activity RNA polymerase II distal enhancer sequence-specific DNA binding

ENA polymerase 1 transcription factor binding transcription factor binding nuclear hormone receptor binding hormone receptor binding

transcription factor activity, direct ligand regulated sequence-specific DNA binding single-stranded DNA binding
histone deacetylase binding steroid hormone receptor activity pre-mRNA binding bHLH transcription factor binding poly-pyrimidin RNA binding poly-pyymidine tract binding
promoter-specific chromatin binding epressing transcription factor binding activating transcription factor bindin distal enhancer DNA-binding transcription activator activity anscription factor activity, RNA polymerase II core promoter sequence-specific DNA binding distal enhancer DNA-binding transcription repressor activity, RNA polymerase II-specific eukaryotic initiation factor $4 \mathrm{E}$ binding mitogen-activated protein kinase kinase kinase binding

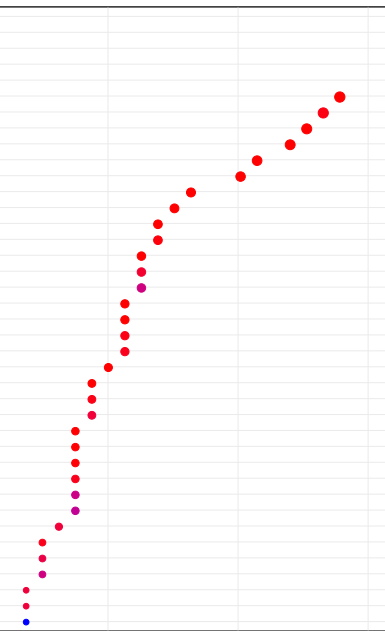



